\title{
Tumor del cuerpo calloso como presentación de neurofibromatosis tipo 1 en un paciente y revisión de la bibliografía
}

\author{
Ignacio Pascual-Castroviejo, Samuel Ignacio Pascual-Pascual, Ramón Velazquez-Fragua, Juan Viaño
}

Servicio de Neurología Pediátrica; Hospital Universitario La Paz (I. Pascual-Castroviejo, S.I. PascualPascual, R. Velazquez-Fraqua) Unidad de Neuroimagen; Hospital del Rosario (J. Viaño); Madrid

España.

Correspondencia: Dr. Ignacio Pascual Castroviejo. Orense, 14, 10. E. E-28020 Madrid.

E-mail:

i.pcastroviejo@

neurologia.e.telefonica.net

Aceptado tras revisión externa:

17.09.12.

Cómo citar este artículo: Pascual-Castroviejo I, PascualPascual SI, Velázquez-Fraqua $\mathrm{R}$ Viaño J. Tumor del cuerpo calloso como presentación de neurofibromatosis tipo 1 en un paciente y revisión de la bibliografía. Rev Neurol 2012; 55: 528-32.

(c) 2012 Revista de Neurología

Introducción. La neurofibromatosis tipo 1 (NF1) es uno de los síndromes neurocutáneos más frecuentes y puede asociarse a tumores intracraneales en cualquier localización, pero excepcionalmente en el cuerpo calloso.

Objetivos. Presentar un caso de NF1 que se manifiesta como un tumor de cuerpo calloso y llevar a cabo una revisión de la incidencia de tumores del cuerpo calloso en nuestra casuística y en la bibliografía.

Caso clínico. Niño visto desde los 3 años con criterios diagnósticos de NF1 (sin comprobación genética), que fue estudiado por resonancia magnética (RM) y RM espectroscópica. La RM mostró objetos brillantes en la neurofibromatosis en diversas zonas cerebrales y cerebelosas, posible tumor en el tronco cerebral (parte bulbar) y tumor en la zona derecha del esplenio del cuerpo calloso. La RM espectroscópica del posible tumor del tronco mostró hallazgos compatibles con tumoración glial de bajo grado. Se siguió su evolución hasta los 19 años sin que hubieran existido cambios clínicos ni en el tamaño de los tumores en las dos localizaciones. Sólo existen seis casos publicados de tumor del cuerpo calloso en pacientes con NF1.

Conclusión. Presentamos un nuevo caso de un paciente con tumor del cuerpo calloso en un paciente con NF1. Las características de la imagen y la evolución clínica van a favor del carácter benigno de este tipo de tumor.

Palabras clave. Neurofibromatosis tipo 1. NF1. Objetos brillantes en la neurofibromatosis. Tumores del cuerpo calloso. Tumores del tronco cerebral. Tumores intracraneales.

\section{Introducción}

La neurofibromatosis tipo 1 (NF1) (OMIM n. $\left.{ }^{0} 162200\right)$ es uno de los síndromes neurocutáneos más frecuentes, que afectan a 1 de cada 3.000 a 3.500 niños [1]. La NF1 es un trastorno multisistémico que puede alcanzar a cualquier órgano del cuerpo, especialmente a estructuras derivadas del ectodermo - piel y sistema nervioso-. Este trastorno está causado por una mutación en el cromosoma 17q11.2, lugar en el que se codifica la neurofibromina, cuyo defecto se relaciona con el origen de los tumores benignos y malignos del sistema nervioso [2]. La NF1 es heredada por vía autosómica dominante a través del defecto cromosómico ya señalado en el $50 \%$ de los casos, y por mutaciones de novo en el otro $50 \%$. El diagnóstico de la NF1 se hace de acuerdo con los criterios acordados en el National Institute of Health Consensus Development Conference Statement [3], siendo necesaria la reunión de al menos dos de los seis criterios acordados para que un paciente sea diagnosticado de NF1.

La presencia de tumores intracraneales en personas con NF1 es de muy diversa localización. Hay zonas muy predispuestas a presentar tumor, como las vías ópticas -nervios ópticos, quiasma, cintillas y proyecciones posteriores-, que comprenden el 14-15\% de los tumores de cualquier localización asociados a NF1 [4-6], mientras que otras localizaciones son bastante infrecuentes, como ocurre con los tumores de los hemisferios cerebrales y la fosa posterior (excluyendo el cerebelo), que constituyen el 1 [7] y el 0,83\% [8], respectivamente. Los tumores del tronco cerebral se sitúan en prevalencias próximas al $4 \%$ [9].

Sin embargo, la revisión de la bibliografía y de nuestra propia casuística nos ha permitido conocer que los tumores intracraneales más infrecuentes son los que se localizan en el cuerpo calloso, raros en la población normal y excepcionales asociados con la NF1. Una revisión de los casos que figuran en nuestra serie de más de 600 pacientes con NF1 (la mayoría, niños menores de 15 años) nos ha permitido encontrar sólo dos casos con tumor en alguna de las zonas que constituyen el cuerpo calloso: uno se localizaba en la zona medial derecha de la porción anterior media del cuerpo calloso, que ha sido ya publicado [10]; y el otro es el caso que presenta- 
Figura 1. Resonancia magnética a los 10 años. Corte sagital con contraste. Se observa ensanchamiento global del bulbo con abundantes imágenes de hiperseñal en el bulbo, en la parte superior del cerebelo y en el hemisferio cerebral. Ensanchamiento global del cuerpo calloso, especialmente en el esplenio.

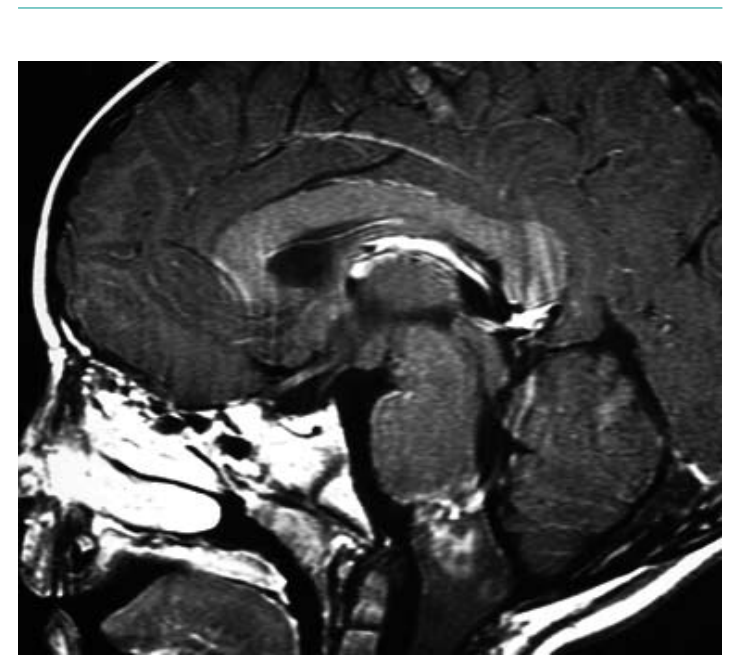

mos en este trabajo, que fue estudiado por padecer otra patología a la que se prestó mayor atención, pasando casi desapercibido el tumor en el esplenio durante varios años debido a la falta de sintomatología clínica, pese a que las imágenes de resonancia magnética (RM) eran incuestionables desde los primeros años de vida.

\section{Caso clínico}

Se trata de un varón, que fue visto por primera vez en el servicio de neurología pediátrica del Hospital Universitario La Paz de Madrid a los 3 años de vida por presentar signos de NF1 asociados con zonas de hiperseñal en el estudio de $\mathrm{RM}$ en secuencia $\mathrm{T}_{2}$ en diversas zonas cerebrales y una imagen circunscrita en la parte derecha del esplenio del cuerpo calloso. El niño había nacido de gestación normal y parto a término, sin problemas, con un peso de 3.100 g y Apgar de 9/10. Su desarrollo psicomotor fue normal, realizando marcha independiente a los 14 meses e iniciando el lenguaje al año. Desde los primeros meses de vida le observaron abundantes manchas café con leche por todo el cuerpo, incluidas axilas e ingles. Ninguno de sus progenitores -padre de 37 años y madre de 35 años- presentaban signos de NF1, y ambos disfrutaban de buena salud.
Figura 2. Resonancia magnética espectroscópica a los 10 años. El espectrograma muestra gran aumento del inositol (Ino), leve incremento de la colina (Co) respecto a la creatina $(\mathrm{Cr})$, y gran disminución del ácido neuroamínico (NAA).

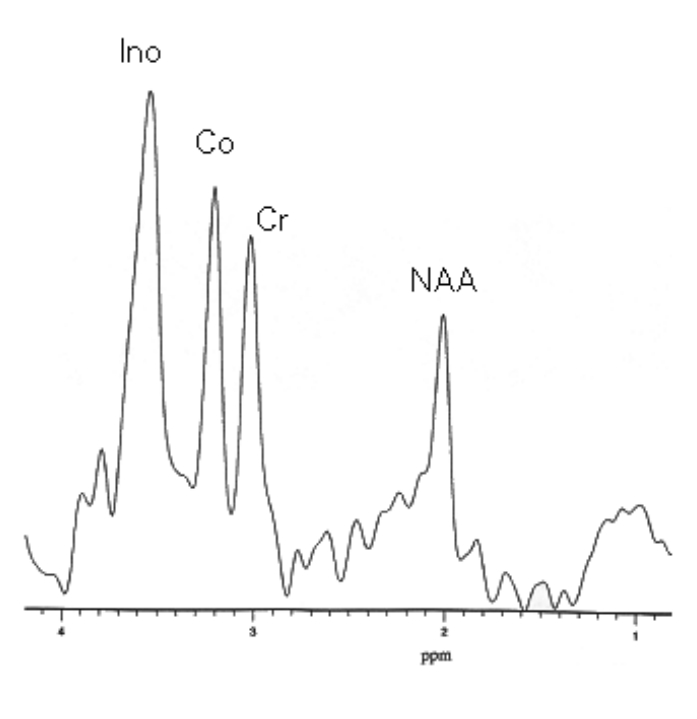

La exploración neurológica mostraba un perímetro cefálico de $53 \mathrm{~cm}$ (percentil + 2 desviaciones estándares) y múltiples manchas café con leche de más de $0,5 \mathrm{~cm}^{2}$ de longitud distribuidas por la piel de todo el cuerpo, presentando también abundantes efélides en ambas regiones inguinales y axilares, cumpliendo de esta forma los dos criterios diagnósticos mínimos de NF1 [3]. Los reflejos miotáticos, pares craneales y pruebas cerebelosas eran normales. El estudio con lámpara de hendidura, que se le practicó posteriormente (a los 10 años), mostró abundantes nódulos de Lisch en el iris de ambos ojos. A los 3 años, la RM cerebral $\left(\mathrm{T}_{1}, \mathrm{~T}_{2}\right.$ y con contraste) mostraba imágenes de hiperseñal distribuidas por las zonas internas de ambos hemisferios cerebrales y en el cerebelo del tipo de los objetos brillantes en la neurofibromatosis, e imágenes patológicas tanto en el tronco cerebral como en el esplenio del cuerpo calloso desde los 3 años de vida.

El estudio del tronco cerebral mostraba hiperseñal irregular extendida a lo largo y a lo ancho del bulbo, y un considerable aumento del grosor del tronco cerebral (Fig. 1). El estudio selectivo de RM espectroscópica del tumor del tronco, practicado posteriormente -a los 10 años-, empleando secuencia de doble eco de espín con tiempo de eco de $35 \mathrm{~ms}$, estaba dominado por la señal del marcador glial inositol y leve incremento de la colina en su relación con la creatina, lo que indicaba leve actividad proliferativa 
Figura 3. Resonancia magnética a los 3 años. Plano coronal en FLAIR, $\mathrm{T}_{2}$ y con contraste, mostrando zonas de hiperseñal en el tronco cerebral, así como la presencia de un tumor redondeado y con zonas de hiperseñal e hiposeñal en la parte derecha del esplenio (flecha).

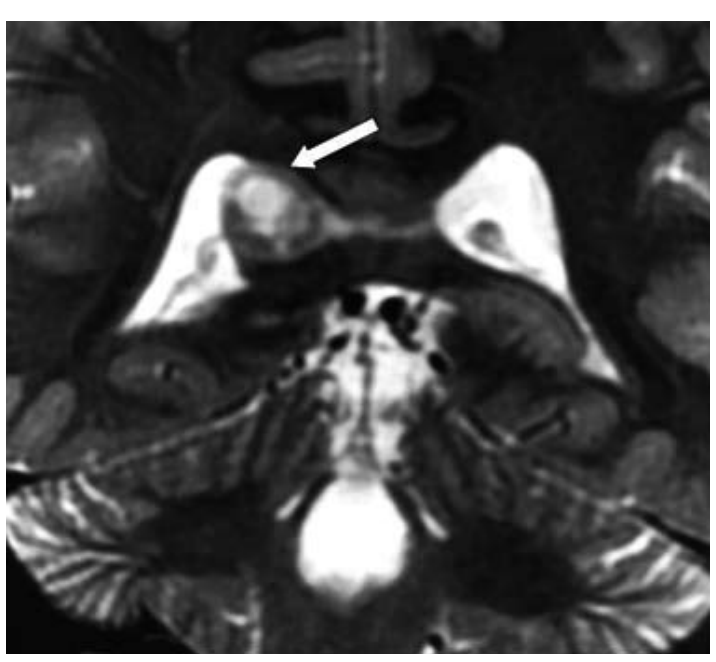

de esta lesión. El marcador neuronal ácido neuroamínico se encontraba significativamente disminuido (Fig. 2), indicando la existencia de daño neuroaxonal en la lesión. Los datos fueron interpretados como correspondientes a lesión en el tronco cerebral, compatibles con tumoración glial de bajo grado, sin signos de agresividad, con pérdida neuroaxonal compatible con larga evolución y leve actividad proliferativa. A los 14 años (cuatro años más tarde que el estudio anterior) se le repitieron todos los estudios de imagen, incluida RM espectroscópica, que mostraron resultados similares a los encontrados cuatro años antes, sin que hubieran disminuido ni el ensanchamiento ni el grado de hiperseñal en el tronco. La lesión en la zona derecha del esplenio del cuerpo calloso aparecía en los primeros estudios de RM, al mismo tiempo que se diagnosticó el tumor en el tronco cerebral. Aparecía como una lesión redondeada en la parte derecha del esplenio, hipointensa en $\mathrm{T}_{1}$ e hiperintensa en $T_{2}$, de unos $2 \mathrm{~cm}$ de diámetro, asociada a un ensanchamiento global del esplenio, especialmente acusado en su parte derecha (Fig. 3). Se repitió el estudio de RM cerebral cada 2-3 años (al mismo tiempo que se seguía la evolución del tumor de tronco), el último a los 19 años, y se observaron imágenes similares a las que se habían obtenido en los estudios anteriores (Fig. 4).

La evolución neurológica del paciente siguió un curso normal, con aptitudes psicomotrices simila-
Figura 4. Resonancia magnética a los 10 años. El corte axial en $\mathrm{T}_{1}$ con contraste muestra hiperseñalizado el tumor en la zona derecha del esplenio (flecha), con tamaño similar al que se veía a los 3 años.

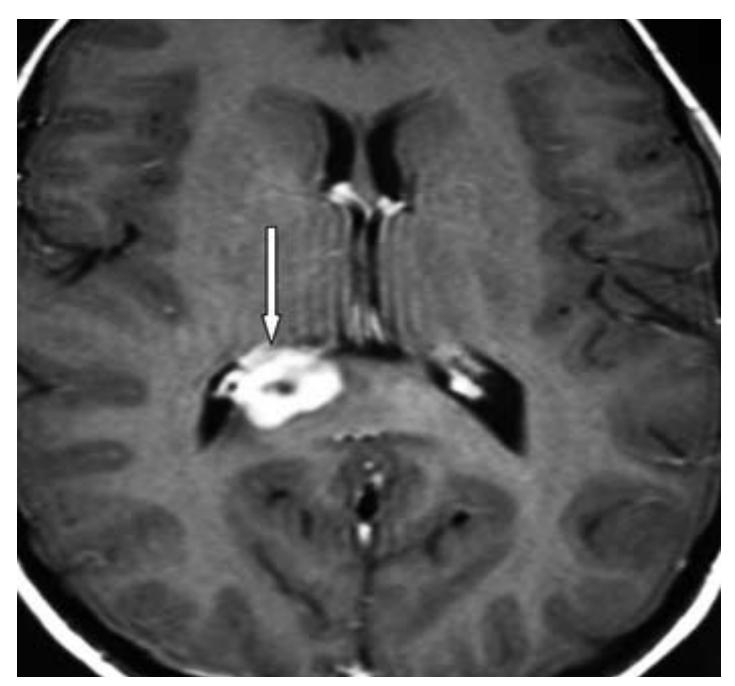

res a las de los niños de su edad y rendimiento escolar normal. Acabó los estudios secundarios a los 15 años, sin perder ningún curso y haciendo deporte (kárate), con un comportamiento normal. En el último control, cuando el paciente tenía 19 años, estaba preparando el acceso a la universidad.

\section{Discusión}

Los tumores del tronco cerebral constituyen casi el $4 \%$ de los tumores intracraneales en pacientes con NF1 [9]. Su prevalencia sólo es superada por la de los tumores de las vías ópticas, que se observan en un 14-15\% [4-6]. La mayor parte de los tumores del tronco cerebral asociados con NF1 tiene carácter histológico y evolutivo benigno [11-13]. Los estudios de RM con contraste muestran la localización y morfología de los tumores del tronco, y la posibilidad de estudios repetitivos es un importante indicador del pronóstico $[14,15]$. La RM espectroscópica también resulta una ayuda para el conocimiento de la posible agresividad y capacidad del crecimiento del tumor [9].

En cualquier caso, las alteraciones recogidas en nuestro paciente no son concluyentes $y$, aunque no pueden catalogarse como normales y se consideraron como demostrativos de corresponder a un proceso expansivo crónico y benigno, su naturaleza his- 
tológica no llegó a aclararse por la buena evolución del niño. Tampoco existen descripciones con comprobación histológica en la bibliografía. De cualquier forma, los casos de malignización son infrecuentes y su mayor peligro para la evolución lo suelen constituir sus altas probabilidades de bloqueo del acueducto y su tendencia al crecimiento hacia los hemisferios cerebelosos siguiendo las vías de sus pedúnculos $[8,9]$.

Entre los tumores intracraneales más infrecuentes asociados con la NF1 están los localizados en el cuerpo calloso. Sólo tenemos constancia de la publicación de seis pacientes previos a éste. El primer caso encontrado en la bibliografía fue aportado por Griffiths et al [16], quienes describían un astrocitoma tipo II, localizado en el esplenio y diagnosticado por biopsia durante un estudio de prevalencia de objetos brillantes en la neurofibromatosis. Anteriormente, Mimouni-Bloch et al [17] publicaron una serie con cuatro pacientes, uno de los cuales tenía confirmación histológica -tras operación- correspondiente a ganglioglioma. Los cuatro pacientes, todas mujeres con edades entre 8 y 19 años, presentaban los tumores en el esplenio, que eran de pequeño tamaño $(0,5 \times 1,5 \mathrm{~cm})$ y apenas crecieron a lo largo del tiempo, observándose el máximo crecimiento en una paciente en la que, tras 16 años de seguimiento, el tamaño del tumor pasó de 1,5 $\times 0,5 \mathrm{~cm}$ a $3,0 \times$ $1,6 \mathrm{~cm}$, sin causar sintomatología clínica. En dos de las pacientes, existían también imágenes tumorales de pequeño tamaño en la rodilla y el cuerpo del cuerpo calloso en una y sólo en la rodilla en otra, que no crecieron a lo largo de los años. Tres de las pacientes mostraban inteligencia normal (incluida la paciente operada) y sólo una, moderada disminución intelectual. Existía trastorno por déficit de atención/hiperactividad (TDAH) en una de las pacientes. El otro caso de tumor del cuerpo calloso descrito [10] estaba originado en la parte medial anterior del cuerpo calloso y crecía hacia el hemisferio cerebral derecho, expandiéndose por el interior del ventrículo lateral derecho, presentando poca afectación neurológica (rendimiento escolar levemente bajo y signos moderados de TDAH). En el paciente descrito en este trabajo no había signos de TDAH.

Los tumores intracraneales asociados con la NF1 se describen a menudo como astrocitomas pilocíticos, tumores que raras veces evolucionan hacia la malignización [18-20]. La mayoría de los tumores de vías ópticas asociados a NF1 presenta este tipo histológico [4-6]. Los casos descritos en la bibliografía de tumores intracerebrales asociados a NF1 hacen pensar que su localización en el cuerpo calloso es escasa, que se localizan preferentemente en el esplenio, y que tienen una escasa o nula repercusión clínica y un crecimiento poco o nada significativo, que no hace necesario el tratamiento farmacológico ni quirúrgico.

En los últimos años se han relacionado con la NF1, aunque sea al menos para tenerlos en consideración como cuadros con los que es preciso hacer el diagnóstico diferencial, algunos trastornos neurocutáneos tipo NF1-like. Los pacientes con estos cuadros presentan signos correspondientes a NF1 incompleta, a síndrome de Noonan y a síndrome de Legius. En estos síndromes existen signos clínicos comunes, como son las lesiones pigmentarias cutáneas, pero otros diferentes. Así, en el síndrome de Noonan existe comúnmente ptosis palpebral uni o bilateral poco pronunciada, hipertelorismo, hipoplasia facial medial, deformidad de los pabellones auriculares, cuello ancho y corto, malformación cardíaca (predominantemente estenosis pulmonar) y pectus excavatum. Los problemas de aprendizaje son comunes a la NF1 y al síndrome de Noonan. Esta asociación de hechos es conocida como síndrome neurofibromatosis-Noonan (OMIM n. ${ }^{\circ}$ 601231) [21-23], presentando la mayoría de ellos la mutación característica de la NF1, pero se han descrito en algunos pacientes la ocurrencia de PTPN11 (síndrome de Noonan) y la mutación de la NF1 [24,25].

El síndrome de Legius es otro cuadro considerado como NF1-like por compartir alteraciones, en grado menor, con la NF1 [26-29]. En este cuadro no se ha descrito hasta el momento la presencia de nódulos de Lisch, neurofibromas, anormalidades óseas, gliomas ópticos ni neurofibromas malignos de nervios periféricos. Al igual que en el síndrome neurofibromatosis-Noonan, tampoco se han descrito todavía tumores en el cuerpo calloso. El síndrome de Legius (OMIM n. ${ }^{\circ}$ 611431) está causado por mutaciones heterocigóticas en SPRED1 [26].

Los estudios comparativos entre NF1 y síndrome de Legius han mostrado un moderado menor nivel intelectual en los pacientes con NF1 [30].

\section{Bibliografía}

1. Riccardi VM. Neurofibromatosis 1. Vol. 1. Basel: Karger; 1988.

2. Ruggieri M, Upadhyaya M, Di Rocco C, Gabriele A, Pascual-Castroviejo I. Neurofibromatosis type 1 and related disorders. In Ruggieri M, Pascual-Castroviejo I, Di Rocco C, eds. Neurocutaneous disorders. Phakomatoses and hamartoneoplastic syndromes. Wien: Springer; 2008. p. 51-151.

3. National Institute of Health Consensus Development Conference Statement. Neurofibromatosis. Arch Neurol 1988: 45: 575-80.

4. Listernick R, Charrow J, Greenwald MJ, Sterby NB. Optic gliomas in children with neurofibromatosis type 1. J Pediatr 1989; 114: 788-99.

5. Pascual-Castroviejo I, Pascual-Pascual SI, Velázquez-Fragua R, Viaño J, García-Segura JM, Botella MP. Neurofibromatosis 
tipo 1 y gliomas de vías ópticas. Una serie de 80 pacientes. Rev Neurol 2008; 46: 530-6.

6. Li J, Perry A, James D, Gutmann DH. Cancer-related expression profiles in NF1-associated pilocytic astrocytomas. Neurology 2001; 56: 885-90.

7. Pascual-Castroviejo I, Pascual-Pascual SI, Viaño J, VelázquezFragua R, Carceller-Benito F, Gutiérrez-Molina M, et al. Tumores de los hemisferios cerebrales en la neurofibromatosis tipo 1 durante la infancia. Rev Neurol 2010; 50: 453-7.

8. Pascual-Castroviejo I, Pascual-Pascual SI, Viaño J, Carceller F, Gutiérrez-Molina M, Morales C, et al. Posterior fossa tumors in children with neurofibromatosis type 1 (NF1). Child Nerv Syst 2010; 26: 1599-603.

9. Pascual-Castroviejo I, Pascual-Pascual SI, Velázquez-Fragua R, Viaño J, García-Segura JM. Tumores del tronco cerebral asociados con neurofibromatosis tipo 1. Presentación de 20 pacientes. Neurologia 2007; 22: 846-52.

10. Pascual-Castroviejo I, Pascual-Pascual SI. Neurofibromatosis type 1 (NF1) associated with tumor of corpus callosum. Child Nerv Syst 2012; Sep 22 [Epub ahead of print].

11. Milstein JM, Geyer JR, Berger MS, Bleyer WA. Favorable prognosis for brainstem gliomas in neurofibromatosis. J Neurooncol 1989; 7: 367-71.

12. Molloy PT, Bilaniuk LT, Vaughan SN, Needle MN, Liu GT, Zackai EH, et al. Brainstem tumors in patients with neurofibromatosis type 1: a distinct clinical entity. Neurology 1995; 45: 1897-902.

13. Bilaniuk LT, Molloy PT, Zimmerman RA, Phillips PC, Vaughan SN, Liu GT, et al. Neurofibromatosis type 1: brainstem tumors. Neuroradiology 1997; 39: 642-53.

14. Peterman SB, Steiner RE, Byder GM, Thomas DJ, Tobias JS, Younger IR. Nuclear magnetic resonance imaging of brainstem tumors. Neuroradiology 1985; 27: 202-7.

15. Bradac GB, Schorner W, Bender A, Felix R. MRI in the diagnosis of brainstem tumors. Neuroradiology 1985; 27 : 208-13.

16. Griffiths PD, Blaser S, Mukonoweshuro W, Armstrong D, Milo-Manson G, Cheung S. Neurofibromatosis bright objects in children with neurofibromatosis type 1 : a proliferative potential? Pediatrics 1999; 104: e49.

17. Mimouni-Bloch A, Kornreich L, Kaadan W, Steinberg T, Shuper A. Lesions of the corpus callosum in children with neurofibromatosis 1. Pediatr Neurol 2008; 38: 406-10.

18. Gutmann DH, Hedrick NM, Li J, Nagarajan R, Perry A, Watson MA. Comparative gene expression profile analysis of neurofibromatosis 1 associated and sporadic pilocytic astrocytomas. Cancer Res 2002; 62: 2085-91.

19. Idoate MA, Echeveste J. Actualización sobre la biología molecular de los gliomas: hacia una clasificación patomolecular de los gliomas. Rev Neurol 2007; 44: 17-24.

20. Rodríguez FJ, Perry A, Gutmann DH, O’Neill BP, Leonard J, Bryant $S$, et al. Gliomas in neurofibromatosis type 1 : a clinicopathologic study of 100 patients. J Neuropathol Exp Neurol 2008; 67: 240-9.

21. Colley A, Donnai D, Evans DGR. Neurofibromatosis/Noonan phenotype: a variable feature of type 1 neurofibromatosis. Clin Genet 1996; 49: 59-64.

22. Carey JC. Neurofibromatosis-Noonan syndrome. Am J Med Genet 1998; 75: 263-4.

23. De Luca A, Bottielo I, Sarkozy A, Carta C, Neru C, Nellacchio E, et al. NF1 gene mutations represent the major molecular event underlying neurofibromatosis-Noonan syndrome. Am J Hum Genet 2005; 77: 1092-101.

24. Bertola DR, Pereira AC, Passetti F, De Oliveira PS, Messiaen L, Gell BD, et al. Neurofibromatosis-Noonan syndrome: molecular evidence of the concurrence of both disorders in a patient. Am J Med Genet A 2005; 136: 242-5.

25. Thiel C, Wilken M, Zenker M, Sticht H, Fahsols R, Gusek-Schneider GC, et al. Independent NF1 and PTPN11 mutations in a family with neurofibromatosis-Noonan syndrome. Am J Med Genet A 2009: 149: 1263-7.

26. Brems H, Chmara M, Sahbatou M, Denayer E, Taniguchi K, Kato R, et al. Germline loss-of function mutations in SPRED1 cause a neurofibromatosis 1-like phenotype. Nat Genet 2007; 39: $1120-6$

27. Messiaen L, Yao S, Brems H, Callens T, Sathienkij-Kanchai A, Denayer E, et al. Clinical and mutational spectrum of neurofibromatosis type 1-like syndrome. JAMA 2009; 302: 2111-8.

28. Pasmant E, Ballerini P, Lapillonne H, Perot C, Vidaud D, Leverger G, et al. SPRED1 disorder and predisposition to leukemia in children. Blood 2009; 114: 1131.

29. Spurlock G, Bennett E, Chuzhanova N, Thomas N, Jim HP, Side L, et al. SPRED1 mutations (Legius syndrome): another clinical useful genotype for dissecting the neurofibromatosis type 1 phenotype. J Med Genet 2009; 46: 431-7.

30. Denayer E, Descheemaeker MJ, Stewart DR, Keymolen K, Plasschaert E, Ruppert SL, et al. Observations on intelligence and behaviour in 15 patients with Legius syndrome. Am J Med Genet C Semin Med Genet 2011; 157: 123-8.

\section{Corpus callosum tumor as the presenting symptom of neurofibromatosis type 1 in a patient and literature review}

Introduction. Neurofibromatosis type 1 (NF1) is one of the most frequent neurocutaneous syndromes. NF1 can be associated with intracranial tumors in any location, but only rarely in the corpus callosum.

Aims. To describe a case of NF1 presenting as a tumor of the corpus callosum and to carry out a review of the incidence of the tumors of corpus callosum in our series and in the literature.

Case report. We present a child who was studied since 3 years of age because of complete NF1 clinical diagnostic criteria (without genetic study). He was studied by MR and magnetic resonance spectroscopy (MRS). MR study showed neurofibromatosis bright objects distributed over several regions of the cerebral hemispheres and cerebellum, a possible brain stem tumor (bulbar zone) and the splenium of the corpus callosum. The MRS of the brain stem tumor showed changes consistent with a low grade glial tumor. The patient was followed until 19-years of age without demonstrating any changes in the clinical features or the tumor size in both locations Only six cases of corpus callosum tumor in patients with NF1 have been published to date.

Conclusions. We present a new case with tumor of the corpus callosum and NF1. The imaging characteristics and the clinical course were in favour of the benign nature of this type of tumor.

Key words. Brain stem tumors. Corpus callosum tumors. Intracranial tumors. Neurofibromatosis bright objects. Neurofibromatosis type 1. NF1. 